\title{
La Extensión del Parentesco y sus Consecuencias Jurídicas
}

\author{
The extension of Kinship and its Legal Consequences
}

\section{Isaura Arguelles-Azuara ${ }^{a}$, Ignacio Sarmiento-Vargas ${ }^{b}$ Víctor T Tomás-Mariano ${ }^{c}$}

\begin{abstract}
:
Within the world of Law, different types of kinship are recognized that, due to their importance, it is necessary to approach the subject in order to deepen and present in the present study the main consequences that derive from kinship that subject is classified in consanguineous kinship, by affinity, adoption or civil. In the development of the work will understand the rights and obligations of relatives in a straight line upward, downward and collateral, who in turn are obliged to provide food and their remoteness is unknown by relatives to what extent you can request such benefit.
\end{abstract}

Keywords:

Marriage, Concubinage, Relationship, Legal Consequences, Principal Obligations

Resumen:

Dentro del mundo del Derecho, se reconocen diferentes tipos de parentesco que por su importancia es menester abordar acerca del tema para profundizar y dar a conocer en el presente estudio las principales consecuencias que derivan del parentesco dicho tema se encuentra clasificado en parentesco consanguíneo, por afinidad, adopción o civil. En el desarrollo del trabajo se comprenderán los derechos y obligaciones de los parientes en línea recta ascendente, descendente y colaterales, quienes a su vez están obligados a dar alimentos y por su lejanía se desconoce por parte de los parientes hasta que grado se puede solicitar dicha prestación.

\section{Palabras Clave:}

Matrimonio, Concubinato, Parentesco, Consecuencias Jurídicas, Obligaciones Principales

\section{Introducción}

A lo largo de la historia y alrededor del mundo el término familia ha ido cambiando, en algunas culturas, países y lugares del mundo, la familia solo era la que estaba unida por el vínculo de consanguinidad, en la historia del derecho romano que es lo más parecido a nuestro derecho se reconocen tres tipos de parentesco el agnatio o civil, el cognatio o natural y por afinidad y/o matrimonio, que eran las situaciones jurídicas que unían a una persona con otra o a varias personas entre sí. Es menester mencionar la institución del matrimonio ya que sin este vínculo jurídico o su afín el concubinato no existiría el parentesco, figura jurídica de la cual se derivan diversos derechos y obligaciones que con el paso del tiempo se ha ido extendiendo. or su importancia México, dentro de su legislación (Código Civil para el Distrito Federal en su Título Cuarto
Bis relativo a la familia en su Capítulo Único) reconoce a la familia de la cual se deriva el parentesco definiéndola como una institución social, permanente, formada por un grupo de personas, unidas por el matrimonio o el concubinato, por el parentesco consanguíneo, adopción y afinidad, así mismo se reconoce como el fundamento de la sociedad y el estado quien a su vez garantiza la seguridad de la familia y su bienestar, en un ambiente libre de violencia entre los miembros que la conforman, bajo su consentimiento para poder procrear y generar con ello el parentesco en sus diversas clasificaciones, por consanguinidad, por afinidad, por adopción o civil.

Objetivo general: Dar a conocer los grados del parentesco en las líneas rectas ascendentes, descendentes y en línea colateral hasta el cuarto grado, así como las instituciones que lo originan y con ello signar la responsabilidad alimenticia de los parientes más próximos en grado.

\footnotetext{
a Autor de Correspondencia, Profesor de Tiempo Completo de la Universidad Autónoma del Estado de Hidalgo, Escuela Superior de Huejutla, https://orcid.org/0000-0002-6403-5435, Email: gari_arg@hotmail.com - isaura_arguelles7778@uaeh.edu.mx

b Profesor de Tiempo Completo de la Universidad Autónoma del Estado de Hidalgo, Escuela Superior de Huejutla, Email: ignacio_sarmiento@hotmail.com 


\section{Hipótesis}

En diversas investigaciones se ha abordado el tema de la obligación alimenticia que nace del parentesco, pero hoy en día aún sigue siendo difícil el cumplimiento de esta prestación debido a que más del $30 \%$ de las sentencias dictadas en los juzgados civiles (Libro de registro de Gobierno del Juzgado Civil y Familiar del Estado de Hidalgo). a favor de otorgar la pensión no se cumplen, por múltiples factores y uno de ellos es el desconocimiento de los grados en las diversas líneas de parentesco que están obligados legalmente a otorgar dicha prestación, de esta manera podemos preguntarnos si las pensiones alimenticias derivadas del parentesco se demandan en procesos judiciales al pariente siguiente en grado, serían pagaderas en un $100 \%$ cumpliendo con ello de forma loable con esta prestación que por su naturaleza es de carácter urgente, necesaria e imprescindible.

\section{Metodología}

Dentro de la presente investigación se darán a conocer los grados de parentesco que son reconocidos legalmente por las leyes de nuestro país, específicamente lo establecido en la Ley familiar para el Estado de Hidalgo y a través de los datos aportados por las instituciones judiciales (Juzgado Civil y Familiar de Primera Instancia de Huejutla de Reyes, Hidalgo), lograremos determinar si las demandas de pensión alimenticia se ejercitan hasta el cuarto grado; grado de parentesco donde se encuentran los primos hermanos, estableciendo con ello la extensión de la obligación alimentaria.

\section{Matrimonio}

El matrimonio tiene vida dentro del derecho romano, con dos grandes clasificaciones, la conventio in manu y el matrimonio: La conventio in manu era un acto formal por virtud de la cual un hombre adquiría una potestad sobre una mujer, esta era similar a la que ejercía sobre los hijos. El matrimonio era un hecho jurídico y no un acto, se actualizaba cuando un hombre y una mujer convivían con la intención de ser considerados cónyuges, este enlace que buscaba mantener una relación jurídica dentro de la descendencia en primer lugar en el derecho romano era de forma facultativa, lo que permitía al varón tomar a una mujer sin previo consentimiento un acto considerado unilateral ya que se otorgaba a los hombres la facultad de poder convivir sin consolidar la relación de derecho, dentro del segundo supuesto encontramos la semejanza con el concubinato ya que bastaba con tener una relación de hecho con los roles prácticos de un matrimonio sin que este se protocolizara, figura actual del concubinato. Con el paso de los años la figura jurídica del matrimonio ha ido sufriendo diversas modificaciones que han sido contempladas en diversas constituciones del mundo y de nuestro país. En el derecho napoleónico, la sociedad francesa postrevolucionaria, supuso que el matrimonio (art. 7 Constitución Francesa, del 3 de septiembre de 1791) como un contrato civil. - "El Poder legislativo establecerá para todos los habitantes, sin distinción, el modo en que se acreditarán los nacimientos, matrimonios y fallecimientos; designará los oficiales públicos que recibirán y conservarán las actas". solo era un contrato civil, por su parte, el código napoleónico regulo el matrimonio como figura laica, pero no como contrato eso desato un intenso debate en la doctrina francesa. Para la eficacia del matrimonio era indispensable la celebración del mismo ante el oficial del estado civil y la existencia del acta de matrimonio, este código se distancio del matrimonio eclesiástico al regular el divorcio y permitir la terminación del vínculo nupcial en vida de los cónyuges, dando vida a ello a las obligaciones subsecuencias con dicha disolución generada por el parentesco.

En México durante el gobierno de Benito Juárez, se realiza una separación muy importante de la Iglesia y el Estado, el 28 de julio de 1859, el presidente Benito Juárez, expuso los motivos de las leyes de Reforma, subrayó la idea de separar cabalmente al Estado respecto de la Iglesia, delimitando el papel que le corresponde a cada uno y reafirmando las tareas que son inherentes al Estado como la más alta organización de la sociedad y con ello estableció la obligatoriedad de todos los habitantes de la República a inscribirse en el Registro Civil. Institución en la que se comprendía los nacimientos, la adopción y arrogación de personas; los matrimonios, los votos religiosos y los fallecimientos; establecía las bases para la expedición de las actas correspondientes que debían estar a cargo de un oficial del estado civil, en la actualidad la figura del registro civil sigue existiendo la institución encargada de registrar lo relativo al estado civil de las personas aunque en otros dicha institución ha cambiado su nombre y se conocen la actualidad como oficialías del estado familiar, con la misma función que se menciona, es aquí donde se establece el primer vínculo legal que estriba en el parentesco de las familias con la institución del matrimonio, el concubinato y las actas de reconocimiento de los hijos. México actualmente tiene definida la institución del matrimonio como una institución social y permanente, por la cual se establece la unión jurídica de un solo hombre y una sola mujer, que, con igualdad de derechos y obligaciones, originan el nacimiento y estabilidad de una familia, así como la realización de una comunidad de vida plena y responsable, (artículo 8 de la Ley para la Familia del Estado de Hidalgo). Esta figura jurídica se consolida dentro del proceso legal de solemnidad ante la fe del oficial del Registro Civil dentro de ciertos estados de la República mexicana y mientras que para otros se denomina Registro del estado familiar, 
esto, por la pluralidad de leyes con que cuenta nuestro país mexicano siendo en todas sus entidades donde surte los efectos legales el matrimonio generando en un primer momento el parentesco por afinidad.

\section{Concubinato}

El concubinato constituye otra de las figuras jurídicas donde se genera el parentesco por afinidad, consanguinidad adopción o civil, por sus orígenes el concubinato ha sido uno de los problemas morales más importantes que ha tenido el Derecho de Familia, así que, también podemos decir que más que un problema político jurídico 0 de regulación técnica, es fundamentalmente una cuestión de orden moral y para poder lograr integrar una relación del derecho con el concubinato y poder regularlo sería ver algunos aspectos del mismo, esta figura jurídica prevalece en la actualidad ya que las parejas de hoy en día optan por vivir de manera pública, pacifica y continua libres del matrimonio realizando como prueba si su estabilidad como pareja integrantes de una familia les permite procrear sin lazos legales que los obliguen a permanecer dentro del núcleo familiar, esta figura es de suma importancia ya que la ley de la familia para el Estado de Hidalgo marca que debe ser registrada, sin embargo, si el registro no se lleva a cabo surte sus efectos jurídicos dentro de los procesos judiciales para acreditar los derechos y obligaciones de las personas integrantes de familia, en relación al esposo, los hijos, por su parte Ignacio Galindo Garfias, nos da su definición del concubinato y lo define como: "La cohabitación entre hombre y mujer la vida en común más o menos prolongada y permanente, es un hecho lícito, que produce efectos jurídicos, pero requiere que para que el concubinato sea reconocido como tal, que tanto el hombre como la mujer lleven vida en común sin estar casados entre sí y sean libres. Por su parte existen otros autores que por su importancia tienen una opinión mas abstracta del concubinato como Clemente Soto Álvarez, y nos dice que es la unión de un hombre y una mujer no ligados en vínculo matrimonial, simplemente este autor refiere que esta figura jurídica debe para que pueda coexistir las parejas integrantes deben estar libres de matrimonio, en virtud de que si alguno de ellos se encuentra ligado a un matrimonio anterior esta figura jurídica no puede existir, por otro lado Baqueiro y Buenrostro mencionan al concubinato "como la unión libre y duradera entre un hombre y una mujer, que viven y cohabitan como si estuvieran casados, y que puede 0 no producen efectos legales "Derecho de Familia y sucesiones, éste último autor menciona de forma importante sobre las consecuencias jurídicas del concubinato quién no finca responsabilidad para los concubinarios, sin embargo, en la actualidad las leyes mexicanas avalan que el concubinato es la unión de un hombre y una mujer libres de matrimonio, que durante más de tres años de manera pública y constante hacen o hicieron vida en común como si estuvieren casados, (Ley para la Familia del estado de Hidalgo), precisa en este apartado que solo debe existir la situación de hecho para que el concubinato surta sus efectos jurídicos esta misma ley establece dentro de su contexto legal las diversas acciones para elevar al concubinato en su legalidad, dentro del mundo del derecho el concubinato es otra figura jurídica que da origen al parentesco en sus diferentes clasificaciones, por consanguinidad, afinidad, adopción o civil.

\section{Antecedentes del parentesco}

Diversas son las formas en que se ha definido el parentesco a través de los años, en el Derecho Romano por su parte en su estudio (Nizama Valladolid, 2009 ), en la revista jurídica denominada la familia en el Derecho Romano y el ordenamiento normativo actual menciona cinco tipos de parentesco; el parentesco agnatio, cognatio, adfinitas, gentilidad y colaterales.

a. El parentesco agnatio, considerado como parentesco civil, en virtud del cual un conjunto de personas se hallan vinculadas entre sí por el poder o autoridad del jefe de la domus o pater familias.

b. El parentesco cognaticio o natural, vincula a las personas por generación o filiación.

c. El parentesco Adfinitas, es el parentesco que surge entre uno de los cónyuges y los parientes consanguíneos del otro cónyuge, dicho parentesco produce efectos jurídicos como impedimentos para el matrimonio y el derecho de pedir alimentos.

d. El parentesco Gentilidad, es el vínculo de familia que guardan pureza de sangre y de tradición de supremacía social y religiosa.

e. El parentesco en colaterales, se cuentan las generaciones partiendo de uno de los parientes hasta el ascendiente común y se regresa luego desde este último hasta el otro pariente, por ejemplo: los hermanos.

A lo largo de la Historia se buscó la manera de establecer los grados las líneas de parentesco y sus obligaciones para con los ascendientes, descendientes y colaterales mas próximos en grado.

Por otro lado, la Constitución Política de los Estados Unidos Mexicanos de 1917, reguló el parentesco consanguíneo, el parentesco por afinidad en los mimos términos del código civil de 1884, esta ley fue la primera en reglamentar la adopción, pero no dispuso que entre el adoptado y el adoptante existiera algún tipo de parentesco.

En la actualidad se sigue conservando la disposición realizada en el año de 1917, que se decretó en la Constitución Política de los Estados Unidos Mexicanos ya que se reconocen por las leyes actuales el parentesco por consanguinidad, afinidad, adopción o civil, y que se 
adopta en la Legislación para el Estado de Hidalgo en la Ley para la Familia en el título séptimo del parentesco y la filiación capítulo I del parentesco y la filiación en su artículo 148 y 149.

\section{Parentesco}

El parentesco es la fuente principal de relaciones y efectos jurídicos familiares, deber, derechos y obligaciones e impedimentos. El número de personas que están unidas entre sí por lazos de parentesco es considerablemente mayor del número de las que están unidas en matrimonio, unión libre o concubinato. El parentesco es la principal fuente de las obligaciones tal y como lo hemos citado anteriormente y nace de la relación que existe entre un hombre y una mujer que puede consolidarse bajo las premisas citadas en los temas anteriores tal como el matrimonio $y$ el concubinato.

El parentesco como concepto doctrinal es definido como la situación jurídica que vincula a dos o más personas físicas en razón de supuestos de consanguinidad, afinidad y adopción simple, (Pérez Fernández). Es importante contextualizar y hacer mención del parentesco como figura jurídica tiene diversas fuentes de las obligaciones mencionaremos lo que establece la legislación en su esencia donde realiza una importa clasificación en líneas y grados de parentesco donde podemos ubicar a los parientes mas próximos en grado y establecer sus derechos y obligaciones de acuerdo a lo que establece la ley, mismo que se encuentra estipulado en el titulo sexto; del parentesco y de los alimentos, capítulo I; del parentesco en los artículos 365 al 373, en la ley para el estado de hidalgo en el titulo séptimo; del parentesco y la filiación, capítulo I; del parentesco en sus artículos 148 al 161, en el código civil federal está estipulado en su artículo 292.

El parentesco como situación jurídica es un conjunto de efectos y relaciones de derecho de duración indefinida y delimitada por una circunstancia 0 serie de circunstancias, con esto queremos decir que las obligaciones y derechos derivados del parentesco no fenecen con el paso del tiempo la consecuencia jurídica de esta relación trasciende después de la muerte signándole para ello a los parientes mas próximos en grado las responsabilidades que se marcan en la ley.

En la Ley para la familia del estado de Hidalgo en el título séptimo del parentesco y la filiación capítulo I del parentesco y la filiación en su artículo 148 y 149 se mencionan o estipulan tres tipos de parentesco: el parentesco consanguíneo, el parentesco por afinidad y el parentesco civil. a) El parentesco consanguíneo, es el vínculo jurídico que se da entre las personas que descienden de un tronco común o que están unidas bilógicamente, otra figura jurídica que corresponde al parentesco es la adopción plena, en esta el adoptado toma el lugar del hijo biológico 0 consanguíneo del 0 de los adoptantes, esta adopción se puede dar entre tíos y sobrinos.

b) El parentesco por afinidad, es el que surge entre el cónyuge y los parientes del esposo o la esposa y entre ellos mismos, tradicionalmente conocidos como nuera, yerno, consuegro, consuegra, cuñado y cuñada, relación de parentesco que se termina después de la disolución del vínculo matrimonial

c) El parentesco civil es el vínculo jurídico que nace de la adopción, pero en las legislaciones como en la doctrina (Ley para la familia del Estado de Hidalgo), (Pérez Fernández); se mencionan dos tipos de adopción: la adopción simple y la adopción plena, la adopción simple es la relación jurídica entre el adoptado y el adoptante o los adoptantes, la adopción plena tiene lugar entre el adoptado y la familia consanguínea del adoptante o los adoptantes, esta adopción también se da entre personas unidas consanguíneamente que se adoptan entre sí, en esta adopción el adoptado remplaza al hijo biológico de los adoptantes adquiriendo los derechos y obligaciones que este tendría con la familia adoptante y rompiendo todo vínculo con su familia biológica.

\section{Líneas y Grados en el Parentesco}

Para medir el parentesco existen formas, determinar la línea y el grado de parentesco existente entre las personas es importante para conocer la relación y efectos y efectos de derechos entre los parientes, el grado es cada una de las generaciones existentes entre personas que jurídicamente son descendientes de un tronco común. La línea de parentesco es una serie de grados estructurada según en orden de las generaciones. El parentesco más cercano es en línea recta, el de primer grado, es el que se da entre progenitores e hijos, el que se da en línea colateral es el segundo grado, es entre hermanos. La línea se divide en dos que son la línea recta y la línea colateral o transversal.

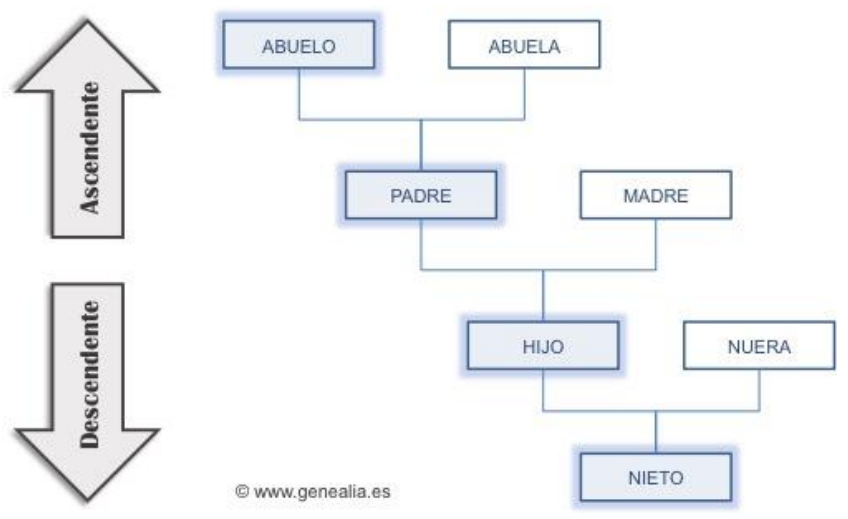


La línea recta se forma por parientes que descienden unos de otros, como los padres e hijos. en este orden se denomina línea recta descendente, por otro lado, tenemos la línea recta ascendente esta última es la que sube donde encontramos a los abuelos, bisabuelos La línea colateral o transversal, es la que se forma por dos líneas rectas que coinciden en un progenitor común, parientes no descendientes unos de otros, pero reconocen un mismo progenitor, hermanos, tíos, sobrinos, primos, etc.

Así mismo la línea colateral o transversal puede ser:

a. Línea colateral o transversa igual, es cuando la distancia de generación que hay entre los parientes de cada línea recta se la misma, los hermanos entre sí y lo primos.

b. La línea colateral o transversal desigual, es cuando la distancia generacional entre los parientes de cada línea recta sea diferente, los tíos, los sobrinos y los primos hermanos.

Quedando los grados tal como se establece en la siguiente tabla, que es de suma importancia para determinar los derechos y obligaciones de los parientes más próximos en grado.

Tabla 1. Parentesco en línea colateral igual, dentro de la siguiente tabla podemos ejemplificar cuales son los parientes próximos en grado y en base a ello identificaremos las responsabilidades de acuerdo a lo que establece la Ley para la familia en el Estado de Hidalgo.

https://www.supercontable.com/informacion/laboral/Grados_

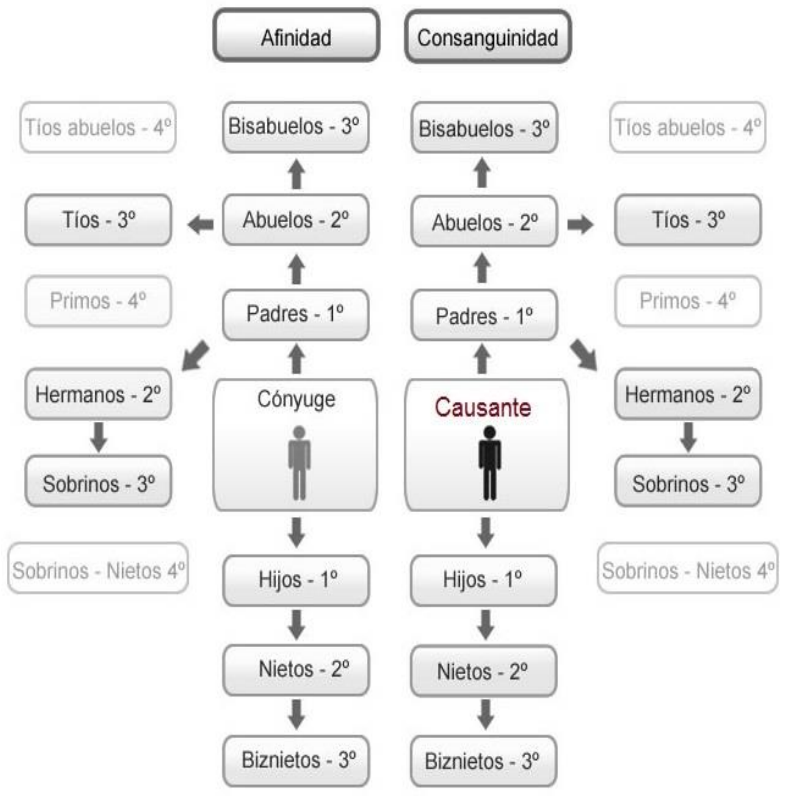

de_Parentesco.html.
Tabla 2. Parentesco en línea recta ascendente y descendente: https://www.genealia.es/grados-de-parentesco/

En la tabla anterior podemos encontrar una ejemplificación del parentesco en línea recta ascendente y descendente donde se signan principalmente los derechos y obligaciones en los miembros de una familia, como pensiones alimenticias y el derecho a suceder dentro de sus principales.

\section{Consecuencias Principales del Parentesco}

El parentesco trae consecuencias que se establecen entre los miembros de la familia, establecidas en la Ley para la Familia del Estado de Hidalgo, en cada uno de los tres tipos de parentesco hay diferentes consecuencias.

En el parentesco por consanguinidad, las relaciones y efectos de este están limitados en algún grado, pero sin duda alguna el parentesco por consanguinidad es uno dentro de sus tres clasificaciones el que aporta obligaciones más amplias, así como los derechos Los efectos personales son:

a. Obligación alimentaria, surge entre los parientes en línea recta hasta el cuarto grado, tal y como se encuentra estipulado en la tabla anterior. (artículo 125 y 27 de la Ley para la Familia del estado de Hidalgo).

b. Patria potestad, solo es entre el menor de edad y sus ascendientes consanguíneos en línea recta hasta el segundo grado.

c. Tutela legitima, esta se da entre los parientes consanguíneos en el cuarto grado.

d. Sucesión legitima, existe entre los parientes consanguíneos en línea recta, sin límite de grado y en línea colateral hasta el cuarto grado.

En el parentesco por afinidad, es un parentesco con menos consecuencias jurídicas, principalmente de impedimentos entre quienes mantienen dicha situación jurídica pero también es considerada para efectos de violencia familiar.

En el parentesco civil, genera los mismos derechos que el parentesco consanguíneo, pero solo entre el adoptante y el adoptado.

Efectos pecuniarios del parentesco: los derechos hereditarios, referido en derecho de sucesión legitima, que solo seda entre el parentesco consanguíneo y el parentesco civil.

El pago de alimentos, esta obligación solo se genera con el parentesco consanguíneo y con el parentesco civil, en el parentesco por afinidad no, porque no existe la obligación con cuñados o cuñadas, además de hacer referencia que el parentesco por afinidad puede 
extinguirse en cualquier momento con la disolución del vínculo matrimonial que lo genera, mientras que los dos anteriores prevalecen aún después de la muerte.(Título cuarto de los alimentos capitulo único de la Ley para la Familia del Estado de Hidalgo).

\section{Alimentos}

Una de las principales consecuencias del parentesco es la obligación alimentaria que nace del vínculo jurídico de esta concepción y se establece entre los parientes más próximos en grado, en ello estriba la importancia de poder ejemplificar cuales son los parientes más próximos en grado y las obligaciones inherentes a cada uno de ellos.

Por su importancia definiremos a los alimentos como, toda asistencia que se presta para el sustento y sobrevivencia de una persona, los alimentos son la comida, el vestido, la habitación y la asistencia en caso de enfermedad (médica o hospitalaria) en estos se incluyen gastos de embarazo y parto. En menores de edad incluye lo anterior, la educación básica y el aprendizaje de un oficio, cuyas características específicas son proporcional, reciproca, a prorrata, indispensable, imprescriptible, inembargable, reciproca, personalísima, intransferible. (título cuarto capítulo único de la Ley para la Familia).

\section{Derecho de Alimentos}

El derecho a alimentos es una obligación que puede satisfacerse de dos formas en virtud de que no solamente la liquidez sino también la incorporación del deudor en la casa del acreedor tal y como lo abordaremos en el tema posterior, como lo establece la Ley para la Familia del Estado de Hidalgo en su artículo 134.

El derecho a alimentos lo puede pedir o reclamar una persona con capacidad de goce y de ejercicio, así como los incapaces a través de un representante, de las personas entre las que señala la ley para su matrimonio y su sobrevivencia, ya que es todo aquello que por ministerio de ley o resolución judicial un individuo (acreedor alimentista) tiene derecho a exigir de otro (deudor alimentista) para vivir, los cuales se encuentran obligados por la figura jurídica del parentesco.

En esta relación está el deudor alimentario, es el sujeto activo del acto jurídico, es la persona que por mandato judicial está obligado a otorgar alimentos y de todo lo que conlleva con ellos.

Ejemplo: vestido, atención médica, etc., los deudores pueden ser el cónyuge, un concubino entre otro.

El acreedor alimentario, es el sujeto pasivo del acto reclamado, es la persona que recibe los alimentos y sus demás accesiones, el acreedor puede ser un hijo, un nieto, cónyuge, concubina, entre otros, tal como lo marca los grados y series según su obligación.

\section{Maneras de Satisfacer una Obligación de Dar}

Existen dos formas de satisfacer la obligación de dar, mediante el pago de la pensión alimenticia, liquida y en dinero, pacto convencional a un proceso judicial, obligación que se cumplirá de padres a hijos, en relación a los cónyuges, con las salvedades que establece la ley. Mediante la incorporación del acreedor alimentista a la casa del deudor, que el que va a recibir los alimentos se vaya a vivir a casa del que los proporcionara, así le daría una casa, alimentos y se tendría que ocupar de la vestimenta, educación hasta que este tenga una profesión y la salud, entre otros, exentando para el parentesco por afinidad cuando los cónyuges son divorciados.

En la satisfacción de alimentos también hay satisfactores especiales como son las siguientes:

a) Gastos de educación y de formación profesional de los menores de edad, los menores de edad tienen derecho a que sus gastos de educación y de formación profesional sean cubiertos por sus deudores alimenticios.

b) Gastos para el desarrollo de las personas discapacitadas o en estado de interdicción, quienes padezcan algún tipo de discapacidad, o hayan sido declarados en estado de interdicción, tienen derecho a que sus deudores alimentarios cubran sus gastos de rehabilitación

c) Gastos para la atención geriátrica de los adultos en edad avanzada, el tercer satisfactor especial de satisfacción son los gastos de atención geriátrica para los adultos en edad avanzada.

\section{Pago de Pensión Alimenticia}

En el pago de esta se engloban: pensión liquida y el dinero, la obligación alimentaria puede cumplirse de dos maneras, mediante la determinación y pago de una pensión o a través de la incorporación del acreedor alimentaria la familia del deudor.

Por asignación de una pensión debe entenderse la determinación de una cantidad periódica de dinero, la cantidad a dar o pagar será dictada por el juez en base a las necesidades del acreedor, por su educación, sus necesidades y en caso de enfermedad el pago de los médicos, medicamentos, estudios médicos y consultas.

Es importante mencionar que las figuras jurídicas que preceden se otorgan a través de un procedimiento judicial, en virtud de que debe existir la acción de la pensión alimenticia y hacerla efectiva en un proceso en las cuales el sujeto obligado a dar alimentos tendría la opción de el pago liquido en dinero o la incorporación del 
acreedor en la casa del deudor alimentista para poder satisfacer la obligación de dar.

Por regla general el deudor alimentario tiene derecho a elegir la manera en que cumplirá la obligación alimentaria.

\section{Incorporación del Acreedor en Casa del Deudor}

La ley para la familia del estado de Hidalgo menciona dos maneras de satisfacer la obligación de dar que por su importancia, se puede concluir que la obligación alimentaria (artículo 134 L.F. E.H.), se cumple por medio de la integración a la familia cuando el deudor alimentario proporciona al acreedor un espacio adecuado para vivir dentro de su casa habitación y sufraga los demás gastos que integran el concepto legal de alimentos, sin embargo, el acreedor carece del derecho de elección cuando el juzgador considera que existe conflicto para la integración del acreedor alimentario a su familia, el órgano jurisdiccional puede negar la incorporación del acreedor de la pensión o alimentos a la casa del deudor - a la familia, en caso de que el obligado hubiera cometido violencia familiar o que el estilo de vida del deudor fuera inconveniente para la estancia o formación del acreedor alimentario, cuando la incorporación sea incompatible con una disposición legal, que el acreedor este sujeto a la patria potestad de persona distinta del obligado, o que el acreedor hubiere sido pareja jurídica del que proporciona los alimentos, de la misma forma podemos mencionar que siempre que exista el indicio que el acreedor alimentista corre riesgo con la incorporación en la casa del deudor se asignara una cantidad líquida en dinero obligatoria para poder satisfacer la obligación de dar alimentos. Por su parte la Ley para la Familia del Estado de Hidalgo maneja dos supuestos para el cumplimiento de dicha obligación

a) Llevar al acreedor alimentario a casa del deudor para proporcionar lo inherente a los alimentos.

b) Proporcionar el cumplimiento de una obligación de forma temporal o permanente.

\section{Sujetos Obligados a Darse Alimentos}

Las personas obligadas a darse alimentos son: (art. 124 de la L.F.E.H.)

I. Los cónyuges excepto;

a) Que el acreedor contraiga nuevas nupcias.

b) Que el acreedor se una en concubinato.

c) Que el acreedor procree un hijo con persona distinta al deudor alimentario.

d) Se demuestre fehacientemente que el ex cónyuge acreedor alimentario cuenta con un empleo mediante el cual perciba una remuneración bastante para satisfacer sus necesidades alimenticias.
II. Los padres, en caso de fallecimiento o imposibilidad para otorgarlos, la obligación recaerá en las siguientes personas: artículo 125 de la Ley para la Familia del Estado de Hidalgo.

a) En los ascendientes por ambas líneas.

b) En los hermanos.

c) En los parientes colaterales hasta el cuarto grado del acreedor alimentario.

III. Los hijos, a falta o por imposibilidad de aquellos, la obligación recaerá en las siguientes personas:

a) A los descendientes más próximos en grado.

b) En los hermanos.

c) En los parientes colaterales hasta el tercer grado del acreedor alimentario, para este caso se requiere el consentimiento de cónyuges del deudor alimentista por sí y en representación de los hijos, (art. 127 y 128 L.F.E.H.).

Un sin número de personas se encuentran obligadas a responder por los menores de edad y por las personas incapacitadas de acuerdo a lo que establecen las normas jurídicas en la extensión del parentesco y los parientes mas próximos en grado, sin embrago, son pocas las personas que conocen las obligaciones que se generan debido al parentesco.

\section{Acciones de pensión alimenticia en el juzgado civil y familiar de primera instancia de Huejutla de Reyes, Hidalgo.}

\begin{tabular}{|l|l|l|l|}
\hline & Ingresos & cumplidas & $\begin{array}{l}\text { Parientes } \\
\text { demandados } \\
\text { próximos en } \\
\text { grado }\end{array}$ \\
\hline 2016 & 700 & $75 \%$ & 0 \\
\hline 2017 & 940 & $80 \%$ & 0 \\
\hline 2018 & 1200 & $65 \%$ & 0 \\
\hline
\end{tabular}

Estadíscas del Juzgado Civil y Familiar de Primera Instancia

De las demandas que se ingresan en el juzgado civil y familiar de primera instancia en un porcentaje significativo no se cumplen a pesar de las medidas de apremio que establece la ley, existiendo factores multifactoriales además del conocimiento por parte de las personas de la obligación que tienen los parientes más próximos en grado, siendo únicamente los autores principales quienes se encuentran demandados para cubrir la pensión alimenticia

\section{Conclusiones}


En el presente trabajo analizamos los derechos y obligaciones de los parientes próximos en grado ya que la obligación alimentaria es una de las principales acciones que prevalece en los juzgados y hemos encontrado en el presente estudio que el parentesco es una figura jurídica que obliga a los parientes más próximos en grado a otorgarse alimentos en caso de que los autores principales no puedan otorgarlos, de esta manera podemos concluir diciendo que la ley protege el interés mayor de los menores de edad extendiéndose ésta obligación el línea recta ascendente y línea recta descendente, así como la colateral, sin embargo, hoy en día en los tribunales no existe acción alguna contra el pariente colateral hasta el cuarto grado y una de las principales causas es el desconocimiento de la obligación alimentaria y su extensión. Por ello podemos concluir dando a conocer que el menor de edad $o$ el incapacitado no debe quedarse sin alimentos de acuerdo a la clasificación y reconocimiento en líneas y grados de parentesco que establece la ley para la Familia del Estado de Hidalgo.

\section{Referencias}

[1] Iglesias Gonzales, Román, Derecho Romano (Cuarta Edición). México, Delegación Cuauhtémoc C.P.06470 México, D.F.: Oxford University

[2] Álvarez, F. R. (2013). Derecho De Familia (Tercera Edición). Av. República Argentina, 15, 06020, México D.F.: Editorial Porrúa.

[3] Asencio, M. F. (2004). La Familia En El Derecho (Quinta Edición). México, Av. República Argentina 15: Porrúa.

[4] Báez, E. B. (2006). Derecho De Familia (Primera Edición). México, Delegación Cuauhtémoc C.P.06470 México, D.F.: Oxford University

[5] Villegas, R. R. (2007). Compendio De Derecho Civil "Introducción, Personas Y Familia", Editorial Porrúa, México.

[6] (S.F.). Obtenido De Los Alimentos En El Derecho Familiar: Https://Repository.Uaeh.Edu.Mx/Revistas/Index.Php/Huejutla/Article/ View/316/312

[7] Biblioteca Jurídica Virtual Del Instituto De Investigaciones Jurídicas de la UNAM. (S.F.). Obtenido de www.juridicas.unam.mx, Http://Biblio.Juridicas.Unam.Mx/Libros/5/2478/4.Pdf

[8] El Parentesco. (S.F.). Obtenido De Bibliotecas Jurídica Virtual Del Instituto de Investigaciones Jurídicas de la UNAM: Https://Archivos.Juridicas.Unam.Mx/Www/Bjv/Libros/7/3270/11.Pdf

[9] Fausto Rico Alvares, P. G. (2013), Derecho De Familia (Tercera Edición Ed.), México, Av. República Argentina, 15: Porrúa S.A. De C.V.

[10] Ley Para La Familia Del Estado De Hidalgo. (S.F.). México.
[11] Villegas, R. R. (2014). Derecho Civil Mexicano 2 Derecho De Familia (Primera Edición Ed.). México: Porrúa S.A. de C.V

[12] Rojina Villegas, Rafael, Compendio De Derecho Civil, Introducción, Personas y Familia, Ed. Porrúa, México, 1984, Pág. 345.

[13] Galindo Garfias. Ignacio, Derecho Civil Primer Curso, Ed.Porrua, Mexico.1985.Pag.480

[14] Soto Alvarez, Clemente., Prontuario De Introducción Al Estudio Del Derecho Civil, Ed. Tercera,Ed. Limusa, México, 1982. Pág. 106.

[15] Https://Www.Gob.Mx/Presidencia/Articulos/Ley-Del-Registro-Civil

[16] Http://Poblacion.Hidalgo.Gob.Mx.

[17] Código Civil para el Distrito Federal en su Título Cuarto Bis relativo a la familia en su Capítulo Único, Pág. 17.

[18] Baqueiro y Buenrostro,’Derecho de Familia y sucesiones. Ed. Oxford. Pag. 121.

[19] Perez Fernández B. (1999), La adopción, Revista Notarial Mexicano Número 113, Pág. 96 y 97.

[20] Nizama Valladolid M. (2009), La Familia en el Derecho Romano y el Ordenamiento Normativo actual, vol. 11, Núm 2-25- 12 ISSN 1817-3594. 